\title{
Accuracy of free hand vs pilot drill and fully guided oral implant placement
}

\author{
Joannis Katsoulis, Prof. Dr.med.dent., MAS'1 \\ Konstantinos Katsoulis, Dr.med. Dr.med.dent.2 \\ 1Department of Reconstructive Dentistry and Gerodontology, School of Dental Medicine, \\ University of Bern, Bern, Switzerland \\ ${ }^{2}$ Department of Maxillofacial Surgery, University hospital, Bern, Switzerland
}

\begin{abstract}
Background
Three-dimensional preoperative implant planning may help to achieve a more predictable postoperative implant position, minimizing anatomical risks and unfavorable prosthodontic outcomes. While different studies investigated the accuracy of computer-assisted implant placement with static CAD/CAM fabricated surgical templates, information on completely free hand and partially guided approaches is limited.
\end{abstract}

\section{Aim/Hypothesis \\ To compare the postoperative implant position with the virtually planned using free hand, pilot drill and fully guided surgical approaches. The hypothesis was that there would be no differences in the accuracy between the 3 surgical protocols.}

\section{Material and Methods}

A controlled in-vitro study with 60 identical maxillary models (3D printed, polilactid) missing the teeth 16, 15, 24, 25, 26 and 27 was performed. The model and tooth setup were digitized with a cone beam computerized tomography (CBCT) and laboratory surface scan, and superimposed using SmartFusion technology. The study coordinator planned the ideal positions for the implants 15, $24(13 / 4.3 \mathrm{~mm})$ and 26 $(8 / 4.3 \mathrm{~mm})$ with the NobelClinician software. Twenty surgeons viewed this ideal planning and placed totally 180 implants using 3 protocols: A) free hand: mentally guided $2 / 3.5 / 4.3 \mathrm{~mm}$ osteotomies (OST) and implant placement (IP), B) pilot drill: template-guided $2 \mathrm{~mm}$ OST (tooth supported NobelGuide template, $2 \mathrm{~mm}$ sleeve) combined with free-hand 3.5/4.3mm OST and IP, C) fully guided: template guided OST and IP (tooth supported NobelGuide template, $2 / 3.5 \mathrm{~mm}$ inserts with $4.3 \mathrm{~mm}$ sleeve). Three-dimensional differences between the digitized postoperative and planned implant positions were measured.

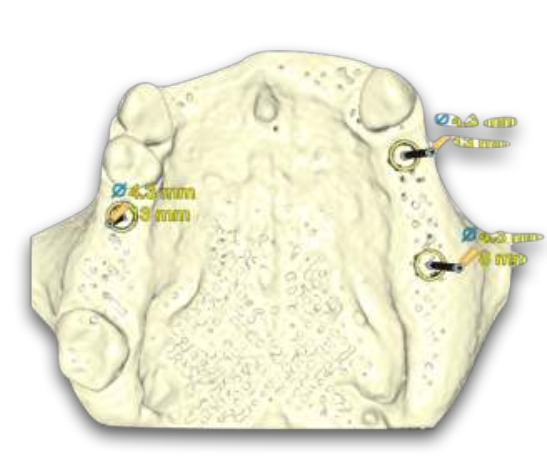

A - free hand 20 models, 60 implants

Osteotomies: $2.0 \mathrm{~mm}$ free han $3.5 \mathrm{~mm}$ free hand $4.3 \mathrm{~mm}$ free hand
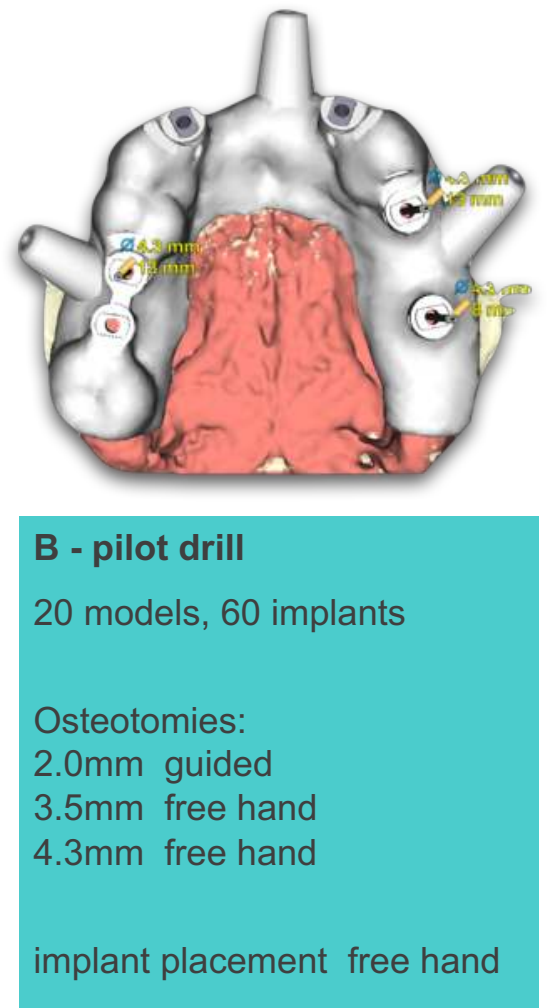
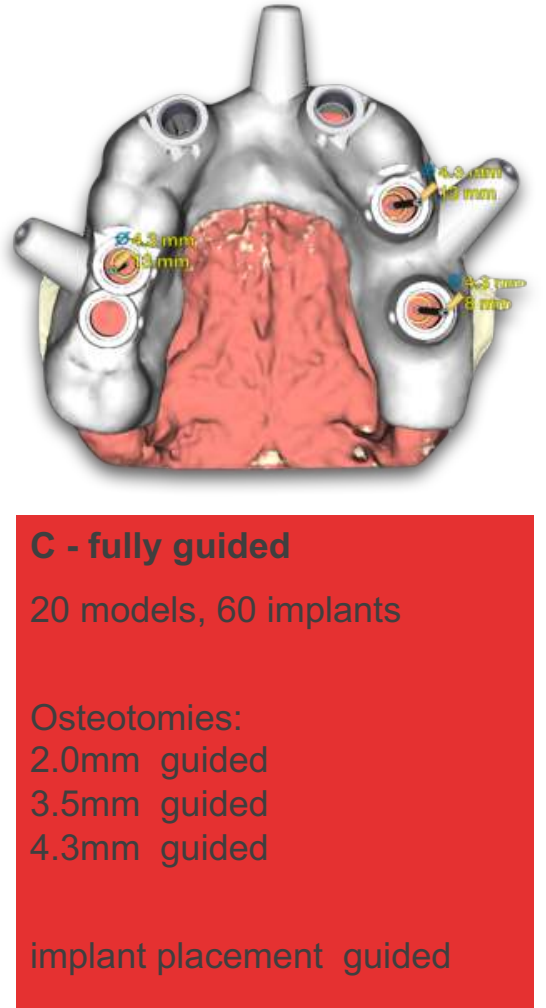

\section{Results}

For the implant protocols A (free hand) vs B (pilot drill) vs C (fully guided) the mean absolute differences $( \pm \mathrm{SD}$; range) between the postoperative and the virtually planned implant positions were $1.1 \mathrm{~mm}( \pm 0.8 ; 0.2-4.7)$ vs $0.7 \mathrm{~mm}( \pm 0.4 ; 0.1-1.8)$ vs $0.5 \mathrm{~mm}( \pm 0.3 ; 0-1.6)$ at the entry point, $1.5 \mathrm{~mm}$ $( \pm 0.7 ; 0.3-3.2)$ vs $1.2 \mathrm{~mm}( \pm 0.6 ; 0.4-2.5)$ vs $0.7 \mathrm{~mm}( \pm 0.4 ; 0.1-2.1)$ at the tip, and $5.1^{\circ}( \pm 4.5 ; 0.1-27.3)$ vs $5.0^{\circ}( \pm 3.2 ; 0.7-19.0)$ vs $1.9^{\circ}( \pm 1.6 ; 0.1-8.6)$ for the angle. Statistical comparisons (ANOVA, Bonferroni correction) of the mean values revealed significant differences at the entry point, i.e. A vs B $(\mathrm{p}<0.001)$, A vs $\mathrm{C}(\mathrm{p}<0.001)$, the tip, i.e. A vs $\mathrm{B}(\mathrm{p}=0.017)$, A vs $\mathrm{C}$ $(p<0.001)$, B vs $C(p<0.001)$, and the angle, i.e. A vs $C(p<0.001)$, B vs C $(\mathrm{p}<0.001)$.
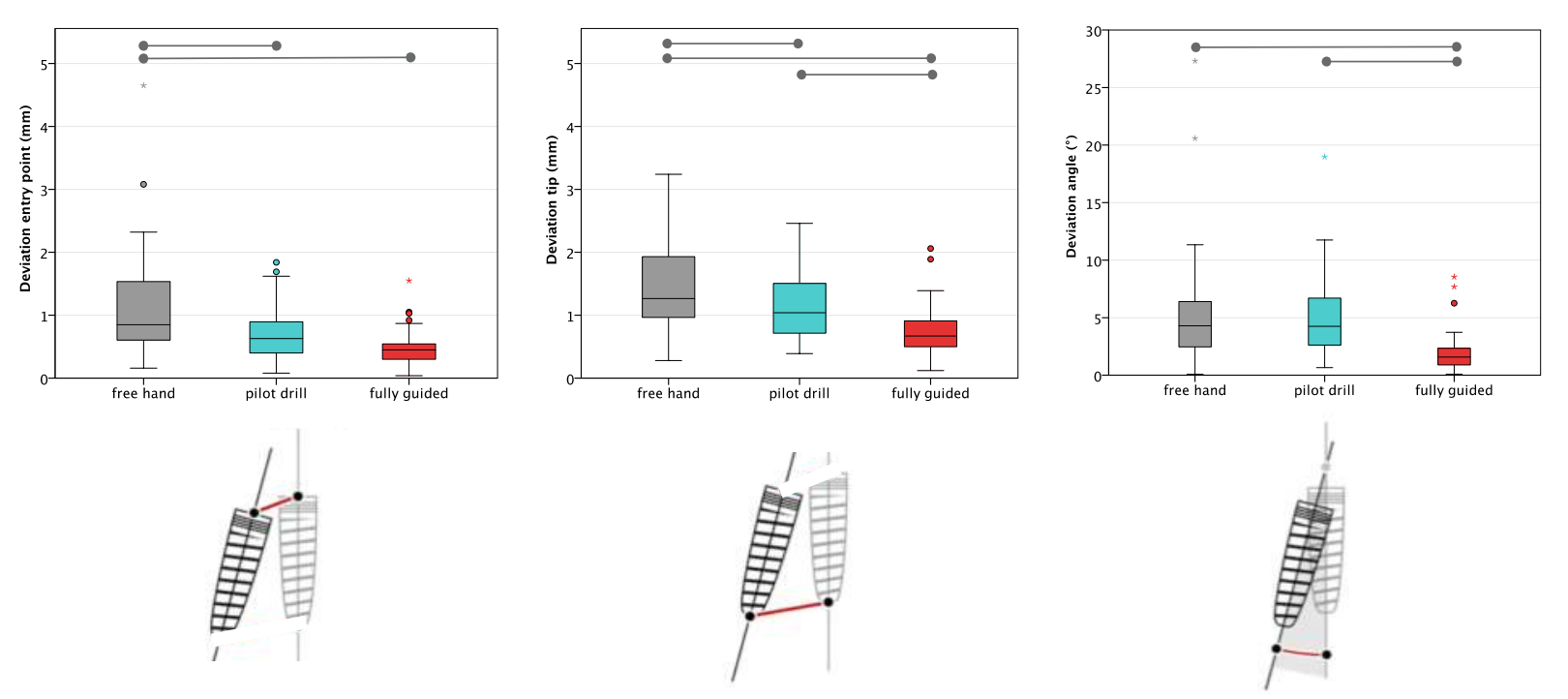

I) Actual implant(postop) $]$ Planned implant (pre-op)

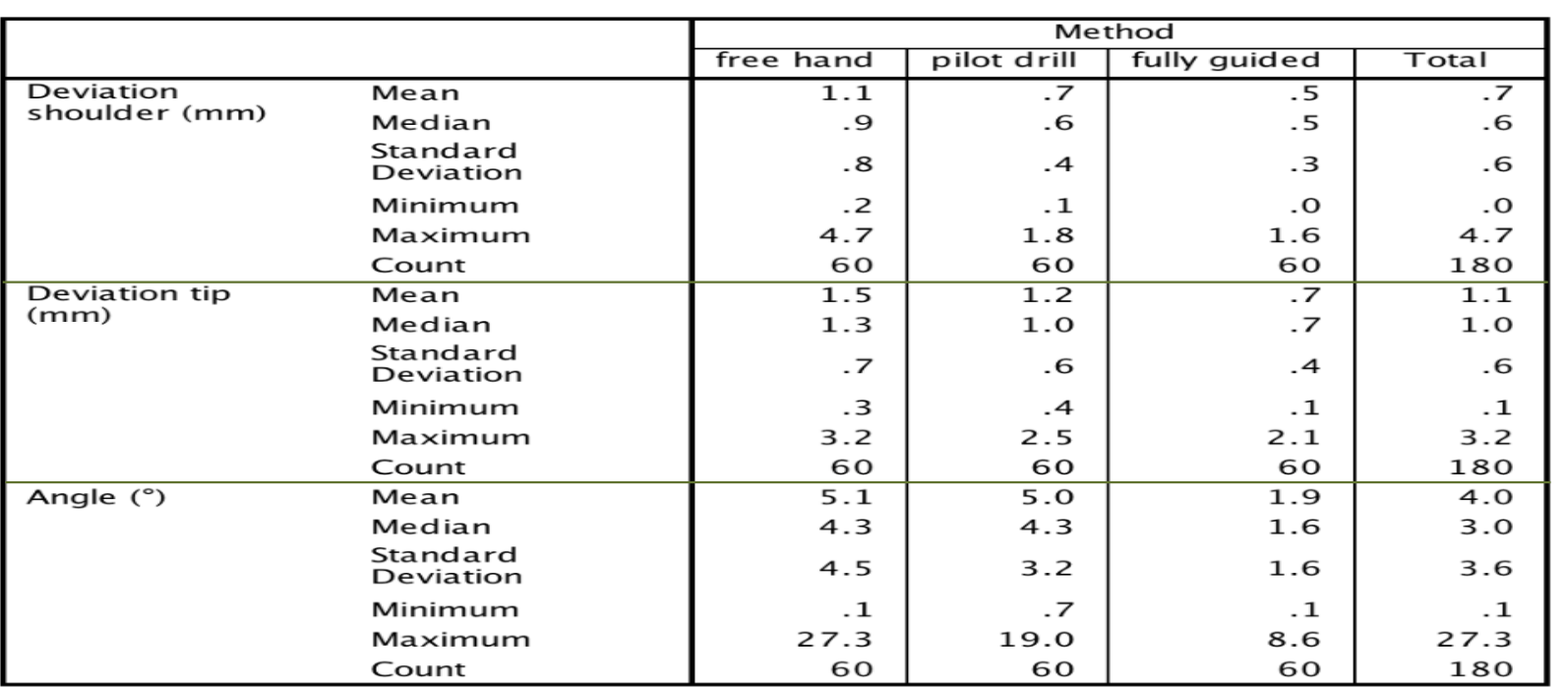

Conclusion and Clinical implications

Mentally guided, free hand osteotomies and implant placement showed significantly less accurate results than the two template guided approaches (factor 1.5-2x). Thus, partially (pilot drill) and especially fully guided implant surgery improve safety and predictability in oral implantology. 